\title{
Congenital Anterolateral Angular Deformity of Tibia
}

\author{
Nareshkumar Satyanarayan Dhaniwalaํ, Khizar Khusrau Khan², Venkatesh Dasari³, Sumit Kumar Baheti ${ }^{4}$ \\ 1, 2, 3, 4 Department of Orthopaedics, Jawaharlal Nehru Medical College, DMIMS, Sawangi, Wardha, Maharashtra, India.
}

\section{INTRODUCTION}

Congenital deformities form a major part of disability burden associated with orthopaedics as most of these go underdiagnosed, untreated or without adequate intervention. Congenital bowing or angular deformities of tibia may be posterior or anterior along with medial or lateral deviation of bone at the apex. Anteromedial bowing with antero-lateral angulation of tibia commonly is associated with incipient or overt pseudarthrosis which can disable a child from the very initial developmental phase of standing and walking. It requires proper follow up, preventive splint and curative treatment which may be prolonged and has guarded prognosis. Due to rarity of the condition the case of bilateral congenital anteromeadial bowing of tibia is being reported.

Congenital angular deformities of the leg may be due to anterior or posterior angulation of tibia with or without fibula angulation. Besides sagittal angulation, tibia is also bowed medially or laterally. Posteromedial deformity of tibia tends to improve with growth. The maximum improvement occurs within $1^{\text {st }}$ year of age which can continue slowly up to the age of 4 years. It is commonly associated with shortening of the bone which may increase till skeletal maturity. Anterior angular deformities of the tibia are more disturbing because these may be precursor to pseudarthrosis of the tibia. Narrowing of the medullary canal or sclerosis at the site of angulation are considered high risk features of pseudarthrosis. Tibia may be deviated medially or laterally along with anterior angulation when viewed from front. Deformity with anterolateral bowing of tibia is more common. Unilateral anterior bowing of the tibia with duplication of the great toe is a separate entity and is considered as its differential diagnosis. Anterior bowing may be associated with shortening of the tibia, clinodactyly and abnormal maturation of carpal bones and metacarpals. ${ }^{1}$

Congenital pseudarthrosis is often associated with anterolateral bowing of tibia, and possesses serious concerns regarding the child's ability or disability status. The condition is reported in 1 in $140000^{2}$ to 1 in 250000 live births. ${ }^{1}$ This case report presents a case of congenital antero-medial bowing of tibia in both the lower limbs in a newborn baby.

\section{PRESENTATION OF CASE}

A $2.1 \mathrm{Kg}$ male child born to $2^{\text {nd }}$ gravida mother at 40 weeks of gestation by elective caesarean section was brought to orthopaedic OPD with deformity and shortening in both the legs. Examination showed anteromedial bowing of both legs at the junction of upper $2 / 3^{\text {rd }}$ and lower $1 / 3^{\text {rd }}$ with dimpling at the site of maximum angulation (Figure 1). The calf muscles, feet and toes were normal. Palpation demonstrated angulation of both tibia and fibula in anterior and medial direction with angulation in anterior and lateral direction. No other bony abnormality was seen in spine, upper limbs, hip and knee. Clinical diagnosis of anterolateral angulation of bilateral tibia and fibula with medial bowing of distal third leg was made.
Corresponding Author: Mr. Khizar Khusrau Khan, Department of Orthopaedics, Jawaharlal Nehru Medical College, DMIMS, Sawangi, Wardha, Maharashtra, India.

E-mail: khizar.khan.khusrau@gmail.com

DOI: $10.14260 / \mathrm{jemds} / 2020 / 787$

How to Cite This Article:

Dhaniwala NS, Khan KK, Dasari V, et al. Congenital anterolateral angular deformity of tibia. J Evolution Med Dent Sci 2020;9(47):3585-3587,

$10.14260 /$ jemds/2020/787

Submission 24-07-2020,

Peer Review 07-10-2020

Acceptance 14-10-2020,

Published 23-11-2020.

Copyright (c) 2020 Nareshkumar Satyanarayan Dhaniwala et al. This is an open access article distributed under Creative Commons Attribution License [Attribution 4.0 International (CC BY 4.0)] 
X-ray of both lower limbs (Figure 2) showed anterior angulation of approximately 60 degree on right tibia and 50 degrees on left tibia. Amount of medial bowing could not be calculated due to x-ray views. There was sclerosis in the posteromedial cortex. Fibula was also having similar deformity on both the sides. Bilateral femur had a mild lateral angulation deformity at the junction of shaft and trochanteric area. X-Rays of upper limbs and skull did not show any abnormality. Vitamin D3 levels of the mother tested to exclude the possibility of congenital rickets, were found normal. Blood investigations- $\mathrm{Hb}-16.2 \%$; $\mathrm{RBC}-5.2$ million / $\mathrm{mm}^{3}$; WBC - $9000 / \mathrm{mm}^{3}$; platelet - 5.1 per microliter; Calcium 9.5 mg / dl; Alkaline phosphatase 9.1 IU / L; T3 - 1.04 ng / dl; T4 $15 \mathrm{ng} / \mathrm{dl}$; TSH - $0.311 \mathrm{~m} \mathrm{IU} / \mathrm{L}$; Vitamin D $30.8 \mathrm{ng} / \mathrm{mL}$ all were within normal limits.

In view of the anterior angulation associated with the sclerosis of the posteromedial part of the tibia, a diagnosis of 'Anteromedial bowing of both legs with incipient congenital pseudarthrosis of tibia-fibula' was considered. The parents were explained the need of long-term bracing of the limb and repeated clinical and radiological follow up to diagnose the pseudarthrosis at the earliest stage. Possibility of surgical treatment was also explained. The child continues under follow up and observation.

\section{DISCUSSION}

Anterior angular deformities are more troublesome due to its association with pseudarthrosis. Posterior angular deformities can get corrected partially with remodelling, but anterior deformities have no spontaneous correction. These keep on increasing with time and develop pseudarthrosis soon without splinting. Even in normal looking tibia any occurrence of narrowing of the medullary canal necessitates splintage till skeletal maturity. ${ }^{1}$ These deformities can be diagnosed by prenatal USG (Ultra-Sono-Graphy) after 20 weeks of gestation. ${ }^{3}$

Congenital pseudarthrosis is a special type of non-union which is either present or incipient at birth. Its exact cause is not known. It is commonly seen in distal half of the tibia, and fibula is also involved in the same limb often. There is a 50 - 90 percent association of this condition with stigmata of neurofibromatosis, including skin and osseous lesions. ${ }^{1}$ There is no spontaneous healing in this condition after fracture without surgical intervention. ${ }^{2}$ This condition may be termed as developmental pseudarthrosis as there is progression after birth and clinical picture of pseudarthrosis is often seen after 1 year of age. Deformity is associated with shortening which may increase as the child grows.

Pseudarthrosis of tibia has been classified based on its radiological features by various authors 4,5, 6 in 4 or 6 subtypes. Anterior angulation of tibia is noted to be a common feature in all classifications. Association of narrowing of medullary canal, presence of a cyst or intraosseous tumour, dysplastic features, sclerosis and thickening of tibial cortex at the site of angulation, defect in tibia with tapering bone ends are other features taken into consideration for classification.

The reported case had anterolateral angulation with medial bowing of both the legs. There was narrowing of medullary canal and the posterior tibial cortex was thickened and sclerosed as seen on x-rays. This case can be classified as type 2 as per Crawford classification. As per Boyd it can be considered as type 1 without a defect in tibia. Its features do not match any type described in Anderson classification.

\section{Boyd Classification of Pseudarthrosis Tibia ${ }^{4}$}

1. Associated with anterior bowing with a defect in tibia.

2. Associated with anterior bowing and hourglass constriction of tibia. The tibia is tapered, rounded and sclerotic with obliterated medullary canal. This type is the most common, often associated with neurofibromatosis and has the poorest prognosis.

3. This develops in a congenital cyst near the junction of distal and middle third of tibia. Anterior bowing may precede or follow a fracture in this case.

4. Develops in a sclerotic segment of bone without narrowing of tibia. The medullary canal may be obliterated.

5. Associated with a dysplastic fibula.

6. Occurs as an intra-osseous neuro fibroma resulting in pseudarthrosis.

\section{Crawford Classification ${ }^{5}$ (Figure 3 )}

1. Has anterolateral bowing with cortical thickening at apex and with open medullary canal.

2. Has narrowed medullary canal and cortical thickening.

3. Has a cystic lesion.

4. Fractured ends of tibia are tapered.

\section{Anderson Classification ${ }^{6}$}

1. Narrow tibia with segmental dysplasia.

2. Cystic tibia with pseudo cysts.

3. Sclerotic and pseudarthrosis appearing at 5 years of age.

4. Anterior tibial bowing with club foot.

Treatment of pseudarthrosis tibia has uncertain prognosis. Treatment modalities vary from correction of angulation and cast application to double only grafting, excision of the local fibrous tissue-bone grafting with stabilization and correction of deformity using ring fixator. Van Nes et al. showed the importance of preventive treatment in the form of casts and brace for curvatures of tibia. ${ }^{7}$ Mother's fibula has been used as a non-vascularized or vascularized graft to help union by many authors. ${ }^{8}$ Some authors have used electromagnetic stimulation of the pseudarthrosis site with uncertain success.

Success of surgical treatment is uncertain due to idiopathic nature of this condition ${ }^{9,10}$ A patient may undergo multiple surgeries before the bones unite. There are post-surgery problems of re-fracture at the pseudarthrosis site, shortening and infection leading to prolonged morbidity. The patient may require to use splints or braces till maturity. Regular clinical and radiological follow up are essential to detect any complications.

Pseudarthrosis tibia treatment still remains a challenge. Regenerative medicine utilizing Platelet Rich Plasma (PRP) may help overcome the problem of pseudarthrosis, as it has been found useful in managing nonunion. ${ }^{11,12}$ Preventive treatment can be done by bridging the curvature by bone grafting or by casting and bracing (supra-patellar orthosis) as supported in a few studies. ${ }^{6,9}$ Lloyd et al. treated 7 patients of 
angular deformity by bone grafting and casting. He observed better results if the grafting is done around 4 years of age. ${ }^{10}$ Congenital anterior bowing of tibia should not be considered similar to congenital posterior bowing of tibia which has a tendency of spontaneous partial correction by remodeling.
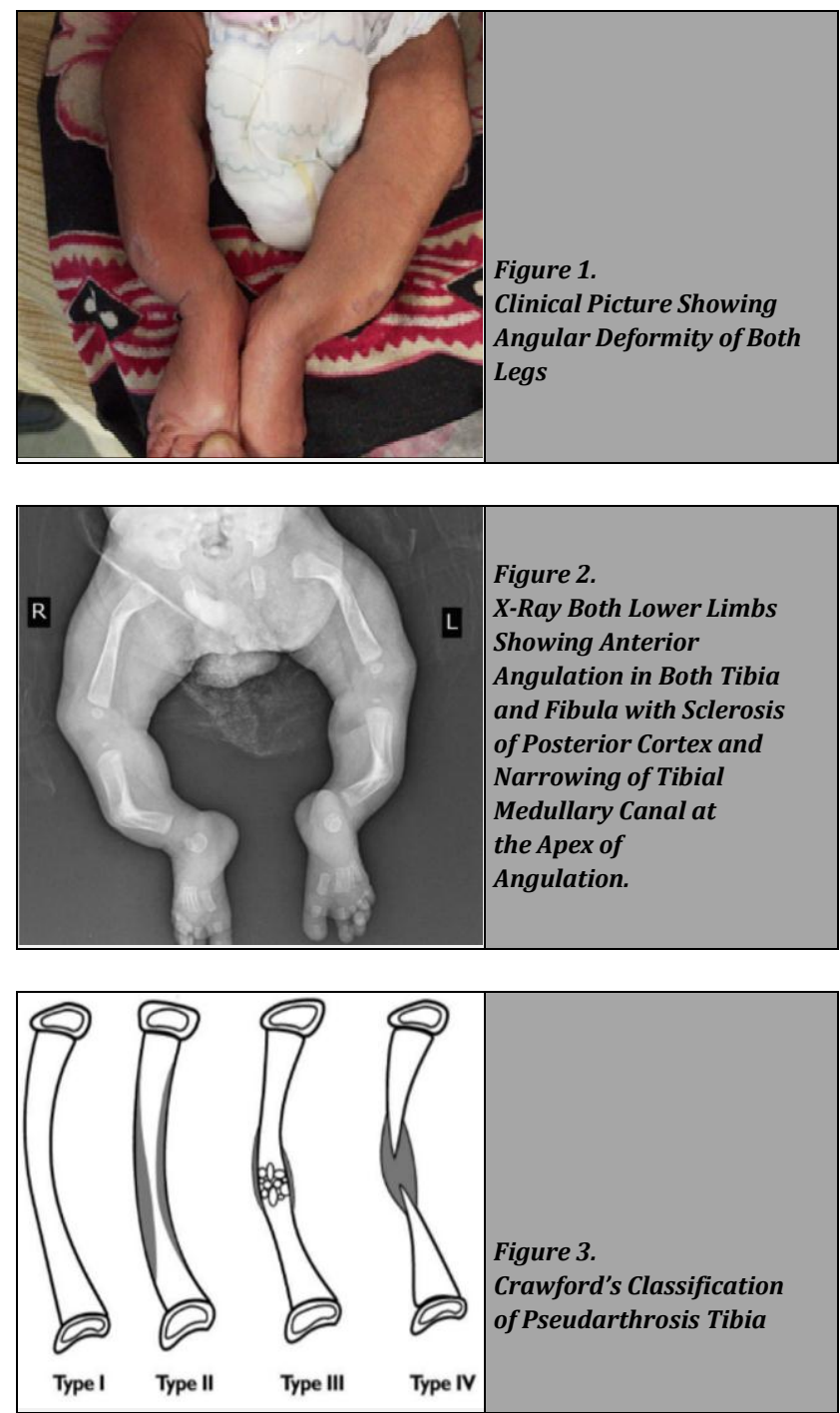

\section{CONCLUSIONS}

Congenital anterior bowing of tibia is considered as incipient pseudarthrosis at the site of angulation. It requires regular follow up, preventive splint and necessary treatment after detection of pseudarthrosis.

Financial or other competing interests: None.

Disclosure forms provided by the authors are available with the full text of this article at jemds.com.

\section{REFERENCES}

[1] Azar FM, Beaty JH, Canale ST. Campbell's operative orthopaedics. Chap-29. Elsevier Health Sci 2017:1053-6.

[2] Weinstein SL, Buckwalter JA, eds. Turek's orthopedics: principles and their application. Lippincott Williams \& Wilkins 2005.

[3] Zollinger PE, Wessels MW, Wladimiroff JW, et al. Prenatal ultrasonographic diagnosis of posteromedial bowing of the leg: two case reports. Ultrasound Obstet Gynecol 2000;15(2):150-3.

[4] Boyd HB. Pathology and natural history of congenital pseudarthrosis of the tibia. Clin Orthop Relat Res 1982;166:5-13.

[5] Crawford AH, Bagamery N. Osseous manifestations of neurofibromatosis in childhood. J Pediatr Orthop 1986;6(1):72-88.

[6] Andersen KS. Congenital pseudarthrosis of the tibia and neurofibromatosis. Acta Orthop Scand 1976;47(1):10811.

[7] Nes CPV. Congenital pseudarthrosis of the leg. J Bone Joint Surg 1966;48(8):1467-83.

[8] Friedman MW, Ritchey CL. Unilateral congenital glaucoma, neurofibromatosis and pseudarthrosis. Arch Ophthalmol 1963;70(3):294-301.

[9] Baker JK, Cain TE, Tullos HS. Intramedullary fixation for congenital pseudarthrosis of the tibia. J Bone Joint Surg Am 1992;74(2):169-78.

[10] Lloyd-Roberts GC, Shaw NE. The prevention of pseudarthrosis in congenital kyphosis of the tibia. J Bone Joint Surg Br 1969;51(1):100-5.

[11] Sharma R, Sharma P, Sharma SD, et al. Platelet-rich fibrin as an aid to soft- and hard-tissue healing. J Maxillofac Oral Surg 2019;28:1-6.

[12] Shrivastava S, Singh PK, Taywade S. Stars therapy: Sandeep's technique for assisted regeneration of skin. J Orthop Allied Sci 2016;4(1):5. 